\title{
Article \\ Definite Integral Involving Rational Functions of Powers and Exponentials Expressed in Terms of the Lerch Function
}

\author{
Robert Reynolds *(D) and Allan Stauffer (D) \\ Department of Mathematics and Statistics, York University, Toronto, ON M3J1P3, Canada; stauffer@yorku.ca \\ * Correspondence: milver@my.yorku.ca
}

\begin{abstract}
This paper gives new integrals related to a class of special functions. This paper also showcases the derivation of definite integrals involving the quotient of functions with powers and the exponential function expressed in terms of the Lerch function and special cases involving fundamental constants. The goal of this paper is to expand upon current tables of definite integrals with the aim of assisting researchers in need of new integral formulae.
\end{abstract}

Keywords: Bose-Einstein; definite integral; Lerch function; Catalan's constant; Glaisher's constant; contour integral

JEL Classification: 30E20; 33-01; 33-03; 33-04; 33-33B; 33E20; 33E33C

check for

updates

Citation: Reynolds, R.; Stauffer, A. Definite Integral Involving Rational Functions of Powers and

Exponentials Expressed in Terms of the Lerch Function.

Math. Comput. Appl. 2021, 26, 58. https://doi.org/10.3390/mca26030058

Received: 29 July 2021

Accepted: 12 August 2021

Published: 18 August 2021

Publisher's Note: MDPI stays neutral with regard to jurisdictional claims in published maps and institutional affiliations.

Copyright: (c) 2021 by the authors. Licensee MDPI, Basel, Switzerland. This article is an open access article distributed under the terms and conditions of the Creative Commons Attribution (CC BY) license (https:/ / creativecommons.org/licenses/by/ $4.0 /)$.

\section{Significance Statement}

The Bose-Einstein integral is defined in Equation (25.12.15) in [1]. This integral is used in a wide range of fields in science and engineering. A few interesting examples are in integrals involving parabolic cylinder functions [2], numerical calculation of a generalized complete elliptic integral [3], the normalization integrals of orthogonal Heun functions [4], the calculation of the stability of repulsive Bose-Einstein condensates in a periodic potential [5], and the generalization of Barton's integral and related integrals of complete elliptic integrals [6].

In this work, the authors look at deriving a generalized form of the Bose-Einstein integral with the aim of expanding current integral tables associated with such research. The hope is that these new tables of definite integrals will aid in expanding potential research requiring such integral formulae. These new integral formulae are derived by using our contour integral method in [7], and a summary of some special cases is itemized in the form of a table for easy reading by potential readers.

\section{Introduction}

With a possible connection to the Bose-Einstein integral, the authors derive the definite integral given by

$$
\int_{0}^{\infty} \frac{e^{m x}(\log (a)+x)^{k}-e^{x(p-m)}(\log (a)-x)^{k}}{e^{p x}-1} d x
$$

where the parameters $k, a$ are general complex numbers and $|\operatorname{Re}(p)|>|\operatorname{Re}(m)|>0$. This definite integral will be used to derive special cases in terms of special functions and fundamental constants. The derivations follow the method used by us in [7]. This method involves using a form of the generalized Cauchy's integral formula given by

$$
\frac{y^{k}}{\Gamma(k+1)}=\frac{1}{2 \pi i} \int_{C} \frac{e^{w y}}{w^{k+1}} d w .
$$

where $C$ is in general an open contour in the complex plane and has the same value at the end points of the contour (see (5.9.2) of Chapter 5 in [1]). When $k$ is not an integer, the 
contour in (2) is an infinite contour around the branch cut that starts at the branch point at $w=0$. We then multiply both sides by a function of $x$ and then take a definite integral of both sides. This yields a definite integral in terms of a contour integral. Then, we multiply both sides of Equation (2) by another function of $x$ and take the infinite sums of both sides such that the contour integral of both equations are the same.

\section{Definite Integral of the Contour Integral}

We use the method in [7]. Using a generalization of Cauchy's integral formula Equation (2), we replace $y \rightarrow x+\log (a)$ and multiply by $e^{m x}$. Next, we form a second equation by replacing $x \rightarrow-x$ and adding both equations and multiplying by $-1 / 2$ in the final form to obtain

$$
-\frac{e^{-m x}(\log (a)-x)^{k}+e^{m x}(\log (a)+x)^{k}}{2 \Gamma(k+1)}=-\frac{1}{2 \pi i} \int_{C} a^{w} w^{-k-1} \cosh (x(m+w)) d w
$$

We repeat the above process except we take the difference between the two derived equations and multiply by $-\frac{1}{2} \operatorname{coth}\left(\frac{p x}{2}\right)$ to obtain

$$
-\frac{\operatorname{coth}\left(\frac{p x}{2}\right)\left(e^{-m x}(\log (a)-x)^{k}-e^{m x}(\log (a)+x)^{k}\right)}{2 \Gamma(k+1)}=\frac{1}{2 \pi i} \int_{C} a^{w} w^{-k-1} \operatorname{coth}\left(\frac{p x}{2}\right) \sinh (x(m+w)) d w
$$

Next, we add Equations (3) and (4), take the definite integral over $x \in[0, \infty)$, and simplify to obtain

$$
\begin{aligned}
& \frac{1}{\Gamma(k+1)} \int_{0}^{\infty} \frac{e^{m x}(\log (a)+x)^{k}-e^{x}(p-m)(\log (a)-x)^{k}}{e^{p x}-1} d x \\
& =-\frac{1}{2 \pi i} \int_{0}^{\infty} \int_{C} a^{w} w^{-k-1}\left(\cosh (x(m+w))-\operatorname{coth}\left(\frac{p x}{2}\right) \sinh (x(m+w))\right) d w d x \\
& =-\frac{1}{2 \pi i} \int_{C} \int_{0}^{\infty} a^{w} w^{-k-1}\left(\cosh (x(m+w))-\operatorname{coth}\left(\frac{p x}{2}\right) \sinh (x(m+w))\right) d x d w \\
& =-\frac{1}{2 \pi i} \int_{C} \frac{\pi a^{w} w^{-k-1} \cot \left(\frac{\pi(m+w)}{p}\right)}{p} d w
\end{aligned}
$$

from Equation (3.411.31) in [8], where $\operatorname{Re}(m+w)>0$ and $|\operatorname{Re}(p)|>|\operatorname{Re}(m)|>0$. When $k$ is not an integer, the terms $\log (a) \pm x$ give branch points and branch cuts which are needed to describe the integral.

The logarithmic function is given, for example, in Section 4.1 in [9]. We are able to switch the order of integration over $\frac{w+m}{p}$ and $x$ using Fubini's theorem since the integrand is of bounded measure over the space $\mathbb{C} \times[0, \infty)$.

\section{The Lerch Function and Infinite Sum of the Contour Integral}

In this section, we use Equation (2) to derive the contour integral representations for the Lerch function.

\subsection{The Lerch Function}

The Lerch function has a series representation given by

$$
\Phi(z, s, v)=\sum_{n=0}^{\infty}(v+n)^{-s} z^{n}
$$

where $|z|<1, v \neq 0,-1, .$. and is continued analytically by its integral representation given by

$$
\Phi(z, s, v)=\frac{1}{\Gamma(s)} \int_{0}^{\infty} \frac{t^{s-1} e^{-v t}}{1-z e^{-t}} d t=\frac{1}{\Gamma(s)} \int_{0}^{\infty} \frac{t^{s-1} e^{-(v-1) t}}{e^{t}-z} d t
$$


where $\operatorname{Re}(v)>0$, and either $|z| \leq 1, z \neq 1, \operatorname{Re}(s)>0$ or $z=1, \operatorname{Re}(s)>1$.

\subsection{Derivation of the First Contour Integral}

Using Equation (2), and replacing $y$ by $\log (a)+\frac{2 i \pi(n+1)}{p}$ and then multiplying both sides by $\frac{2 i \pi}{p} e^{\frac{2 i \pi m(n+1)}{p}}$, taking the infinite sum over $n \in[0, \infty)$ and simplifying in terms of the Lerch function, we obtain

$$
\begin{aligned}
& \frac{(2 \pi)^{k+1}\left(\frac{i}{p}\right)^{k+1} e^{\frac{2 i \pi m}{p}} \Phi\left(e^{\frac{2 i m \pi}{p}},-k, 1-\frac{i p \log (a)}{2 \pi}\right)}{\Gamma(k+1)} \\
& =\frac{1}{2 \pi i} \sum_{n=0}^{\infty} \int_{C} \frac{2 i \pi a^{w} w^{-k-1} e^{\frac{2 i \pi(n+1)(m+w)}{p}}}{p} d w \\
& =\frac{1}{2 \pi i} \int_{C} \sum_{n=0}^{\infty} \frac{2 i \pi a^{w} w^{-k-1} e^{\frac{2 i \pi(n+1)(m+w)}{p}}}{p} d w \\
& =-\frac{1}{2 \pi i} \int_{C} \frac{\pi a^{w} w^{-k-1}\left(\cot \left(\frac{\pi(m+w)}{p}\right)+i\right)}{p} d w
\end{aligned}
$$

from Equation (1.232.1) in [8], where $\operatorname{Im}\left(\frac{w+m}{p}\right)>0$ in order for the sum to converge.

\subsection{Derivation of the Additional Contour}

Using Equation (2), we replace $y \rightarrow \log (a)$ and multiply both sides by $-\frac{i \pi}{p}$ to obtain

$$
\frac{i \pi \log ^{k}(a)}{p \Gamma(k+1)}=\frac{1}{2 \pi i} \int_{C} \frac{i \pi a^{w} w^{-k-1}}{p} d w
$$

\section{Main Results}

In the proceeding section, we will evaluate Equation (10) for various special cases of the parameters $k, a, p$ and $m$. We will simplify the Lerch function in terms of the polylogarithm $L i_{s}(z)=z \Phi(z, s, 1)$ Equation (64:12:2) in [10] with special cases $\Phi(-1,2,1 / 2)=4 G$, where $G=\sum_{j=0}^{\infty} \frac{(-1)^{j}}{(2 j+1)^{2}}$ is Catalan's constant given in Equation (1:7:4) in [10], and the zeta function of Riemann $\zeta(s)=\zeta(s, 1)=\Phi(1, v, 1)$ from Equation (25:14:2) in [1].

Theorem 1. For all $k, a \in \mathbb{C}$ and $|\operatorname{Re}(p)|>|\operatorname{Re}(m)|>0$,

$$
\begin{aligned}
& \int_{0}^{\infty} \frac{e^{m x}(\log (a)+x)^{k}-e^{x(p-m)}(\log (a)-x)^{k}}{e^{p x}-1} d x \\
& =(2 \pi)^{k+1}\left(\frac{i}{p}\right)^{k+1} e^{\frac{2 i \pi m}{p}} \Phi\left(e^{\frac{2 i m \pi}{p}},-k, 1-\frac{i p \log (a)}{2 \pi}\right)+\frac{i \pi \log ^{k}(a)}{p}
\end{aligned}
$$

Proof. Observe that the right-hand side of Equation (5) is equal to the sum of the righthand sides of Equations (8) and (9), so we may equate the left-hand sides and simplify the Gamma function to yield the stated result.

Lemma 1.

$$
\int_{0}^{\infty} \operatorname{csch}\left(\frac{p x}{2}\right) \sinh \left(m x-\frac{p x}{2}\right) d x=-\frac{\pi \cot \left(\frac{\pi m}{p}\right)}{p}
$$

Proof. Use Equation (10), set $k=0$, and simplify using entry (2) in the table below (64:12:7) in [10].

\section{Lemma 2.}

$$
\int_{0}^{\infty} x \operatorname{csch}\left(\frac{p x}{2}\right) \cosh \left(m x-\frac{p x}{2}\right) d x=\frac{\pi^{2} \csc ^{2}\left(\frac{\pi m}{p}\right)}{p^{2}}
$$


Proof. Use Equation (10), set $k=a=1$, and simplify using entry (3) in the table below (64:12:7) in [10]. This is Equation (3.411.31) in [8].

Lemma 3.

$$
\begin{aligned}
& \int_{0}^{\infty} \frac{\log (a)\left(e^{x(p-m)}-e^{m x}\right)+x\left(e^{x(p-m)}+e^{m x}\right)}{\left(e^{p x}-1\right)\left(x^{2}-\log ^{2}(a)\right)} d x \\
& =e^{\frac{2 i \pi m}{p}} \Phi\left(e^{\frac{2 i m \pi}{p}}, 1,1-\frac{i p \log (a)}{2 \pi}\right)+\frac{i \pi}{p \log (a)}
\end{aligned}
$$

Proof. Use Equation (10), set $k=-1$, and simplify.

Lemma 4. For $\operatorname{Re}(k)>0$,

$$
\int_{0}^{\infty} \frac{x^{k} e^{m x}-(-x)^{k} e^{x(p-m)}}{e^{p x}-1} d x=\frac{i(2 \pi)^{k+1}\left(\frac{i}{p}\right)^{k} L i_{-k}\left(e^{\frac{2 i m \pi}{p}}\right)}{p}
$$

Proof. Use Equation (10), set $a=1$, and simplify using Equation (64:12:2) in [10].

\section{Example 1.}

$$
\int_{0}^{\infty} \frac{x \operatorname{csch}(x)}{x^{2}+\pi^{2}} d x=\log (2)-\frac{1}{2}
$$

and

$$
\int_{0}^{\infty} \frac{x^{2} \operatorname{coth}(x) \operatorname{csch}(x)}{x^{2}+\pi^{2}} d x=\frac{1}{12}\left(\pi^{2}-6\right)
$$

Proof. Use Equation (10), take the first partial derivative with respect to $k$, set $k=-1, a=-1$, $p=2, m=1$, and simplify in terms of the real and imaginary parts using entry (1) and entry (2) in the table below (64:12:7) in [10].

\section{Example 2.}

$$
\int_{0}^{\infty} \frac{x \operatorname{csch}(x)}{4 x^{2}+\pi^{2}} d x=\frac{1}{8}(\pi-2)
$$

and

$$
\int_{0}^{\infty} \frac{x^{2} \operatorname{coth}(x) \operatorname{csch}(x)}{4 x^{2}+\pi^{2}} d x=\frac{1}{4}(2 G-1)
$$

Proof. Use equation (10), take the first partial derivative with respect to $k$, set $k=-1$, $a=-1, p=2, m=1$, and simplify in terms of the real and imaginary parts and Catalan's constant, $G$, using entry (1) and entry (2) in the table below (64:12:7) in [10].

Lemma 5. For $\operatorname{Re}(m)>0$,

$$
\int_{0}^{\infty} \arctan \left(\frac{x}{a}\right) \operatorname{csch}(m x) d x=\frac{\pi \log \left(\frac{a m \Gamma\left(\frac{a m}{2 \pi}\right)^{2}}{2 \pi \Gamma\left(\frac{a m+\pi}{2 \pi}\right)^{2}}\right)}{2 m}
$$

Proof. Use Equation (10), set $p=2 m, a=a i$, and simplify in terms of the Hurwitz zeta function $\zeta(s, v)$ using entry $(4)$ in the table below (64:12:7) in [10]. Next, take the first partial derivative with respect to $k$, set $k=0$, and simplify the logarithm on the left-hand side in terms of the arctangent function using Equation (64:10:2) in [10].

Lemma 6. For $\operatorname{Re}(k)>0, \operatorname{Re}(m)>0$,

$$
\int_{0}^{\infty} x^{k} \operatorname{csch}(m x) d x=\left(2^{k+1}-1\right) \pi^{k+1} m\left(\frac{i}{m}\right)^{k+2} \zeta(-k)\left(\cot \left(\frac{\pi k}{2}\right)-i\right)
$$


Proof. Use Equation (10), set $p=2 m$, and simplify in terms of the Hurwitz zeta function $\zeta(s, v)$ using entry (4) in the table below (64;12:7) in [10]. Next, set $a=1$ and simplify in terms of the zeta function of Riemann $\zeta(s)$ using entry (2) in the table below (64:7) in [10].

Lemma 7.

$$
\int_{0}^{\infty} \frac{x^{2} \operatorname{coth}(x) \operatorname{csch}(x)}{a^{2}+x^{2}} d x=\frac{\pi}{2 a}+\frac{a\left(\psi^{(1)}\left(\frac{a}{2 \pi}+1\right)-\psi^{(1)}\left(\frac{a+\pi}{2 \pi}\right)\right)}{4 \pi}
$$

Proof. Use Equation (10), set $p=2 m$, and simplify in terms of the Hurwitz zeta function $\zeta(s, v)$ using entry $(4)$ in the table below $(64 ; 12: 7)$ in [10]. Next, we take the first partial derivative with respect to $m$ and set $m=1$. Next, we apply l'Hôpital rule to the right-hand side as $k \rightarrow-1$, replace $a \rightarrow e^{a}$, and simplify using Equation (64:4:1) in [10].

Lemma 8. For $\operatorname{Re}(m)>0$,

$$
\begin{aligned}
& \int_{0}^{\infty}\left(\left(x+\frac{i \pi}{m}\right)^{k}-\left(-x+\frac{i \pi}{m}\right)^{k}\right) \operatorname{csch}(m x) d x \\
& =\frac{\pi^{k+1}\left(\frac{i}{m}\right)^{k-1}\left(\left(2^{k+2}-2\right) \zeta(-k)+1\right)}{m^{2}}
\end{aligned}
$$

Proof. Use Equation (10), set $a \rightarrow e^{2 \pi i / p}$, and simplify; then, replace $p \rightarrow 2 m$ and simplify in terms of the zeta function of Riemann $\zeta(s)$ using entry (2) in the table below (64:7) and entry (1) in the table below (64:12:7) in [10].

Lemma 9. For $\operatorname{Re}(m)>0$,

$$
\int_{0}^{\infty} \frac{x \operatorname{csch}(m x)}{m^{2} x^{2}+\pi^{2}} d x=\frac{\log (4)-1}{2 m^{2}}
$$

Proof. Use Equation (22), apply l'Hôpital rule to the right-hand as $k \rightarrow-1$, and simplify.

Lemma 10. For $\operatorname{Re}(m)>0$,

$$
\int_{0}^{\infty} \frac{x \operatorname{csch}(m x)}{\left(m^{2} x^{2}+\pi^{2}\right)^{2}} d x=\frac{\pi^{2}-6}{24 \pi^{2} m^{2}}
$$

Proof. Use Equation (22), set $k=-2$, and simplify.

Example 3.

$$
\int_{0}^{\infty} \frac{(\pi-x)(x+\pi) \operatorname{sech}(x)}{\left(x^{2}+\pi^{2}\right)^{2}} d x=\frac{4-4 G}{\pi}
$$

and

$$
\int_{0}^{\infty} \frac{x \operatorname{csch}(x)}{\left(x^{2}+\pi^{2}\right)^{2}} d x=\frac{1}{24}-\frac{1}{4 \pi^{2}}
$$

Proof. Use Equation (10), set $k=-2, a=-1, m=1, p=4$, compare real and imaginary parts, and simplify in terms of Catalan's constant, $G$, using entry (3) in the table below (64:12:7) and Equation (63:13:4) in [10]. 
Lemma 11. For $\operatorname{Re}(m)>0$,

$$
\begin{aligned}
& \int_{0}^{\infty}\left(\frac{\log (\log (a)-x)}{x-\log (a)}+\frac{\log (\log (a)+x)}{\log (a)+x}\right) \operatorname{csch}(m x) d x \\
& =\log \left(\frac{2 i \pi}{m}\right)\left(H_{-} \frac{i m \log (a)+\pi}{2 \pi}-H_{-\frac{i m \log (a)}{2 \pi}}\right)-\gamma_{1}\left(\frac{\pi-i m \log (a)}{2 \pi}\right) \\
& +\gamma_{1}\left(1-\frac{i m \log (a)}{2 \pi}\right)+\frac{i \pi \log (\log (a))}{m \log (a)}
\end{aligned}
$$

Proof. Use Equation (10), set $p \rightarrow 2 m$, and simplify in terms of the Hurwitz zeta function $\zeta(s, v)$. Next, take the first partial derivative with respect to $k$. Next, apply l'Hôpital rule to the right-hand side as $k \rightarrow-1$, simplify using Equation (64:10:2) in [10], and simplify in terms of the Harmonic function $H_{n}$, the Stieltjes constant $\gamma_{n}$, and the generalized Stieltjes constant $\gamma_{n}(a)$ using (3:6:7) in [10] and Equation (1.1) in [11].

\section{Example 4.}

$$
\int_{0}^{\infty} \frac{x \operatorname{csch}(x)}{x^{2}+\pi^{2}} d x=\log (2)-\frac{1}{2}
$$

and

$$
\begin{aligned}
& \int_{0}^{\infty} \frac{\operatorname{csch}(x)\left(x \log \left(x^{2}+\pi^{2}\right)-2 \pi \tan ^{-1}\left(\frac{x}{\pi}\right)\right)}{x^{2}+\pi^{2}} d x \\
& =-\gamma_{1}+\gamma_{1}\left(\frac{3}{2}\right)+\log (\pi)+(\log (4)-2) \log (2 \pi)
\end{aligned}
$$

Proof. Use Equation (27), set $a=-1, m=1$, compare and separate real and imaginary parts, and simplify.

\section{Example 5.}

$$
\begin{aligned}
& \int_{0}^{\infty} e^{x}\left(\frac{4 \log \left(-x+\frac{i \pi}{2}\right)}{(\pi+2 i x)^{2}}+\frac{\log \left(x+\frac{i \pi}{2}\right)}{\left(x+\frac{i \pi}{2}\right)^{2}}\right)(\operatorname{coth}(x)-1) d x \\
& =-\frac{2 i\left(\Phi^{\prime}\left(-1,2, \frac{3}{2}\right)+4 G \log (\pi)-2 \log (2 \pi)\right)}{\pi}+4 G-2
\end{aligned}
$$

Proof. Use Equation (10), take the first partial derivative with respect to $k$, set $k=-2, a=i$, $p=2, m=1$, and simplify in terms of Catalan's constant $G$ using Equation (6) in [12].

\section{Example 6.}

$$
\int_{0}^{\infty} x \operatorname{csch}(x) d x=\frac{\pi^{2}}{4}
$$

and

$$
\begin{aligned}
& \int_{0}^{\infty} \operatorname{csch}(x)\left(x \log \left(x^{2}+\pi^{2}\right)+2 \pi \tan ^{-1}\left(\frac{x}{\pi}\right)\right) d x \\
& =\frac{1}{6} \pi^{2}\left(36 \log (A)-3+\log \left(\frac{\pi^{3}}{16}\right)\right)
\end{aligned}
$$

Proof. Use Equation (10), set $p=2 m$, and simplify in terms of the Hurwitz zeta function $\zeta(s, v)$ using entry $(4)$ in the table below $(64: 12: 7)$ in [10]. Next, we take the first partial derivative with respect to $k$, set $k=1, a=-1, m=1$, and simplify in terms of Glaisher's constant $A$ after expanding in terms of the real and imaginary parts.

\section{Example 7.}

$$
\int_{0}^{\infty} x \operatorname{csch}(x) d x=\frac{\pi^{2}}{4}
$$

and

$$
\int_{0}^{\infty} \operatorname{csch}(x)\left(x \log \left(x^{2}+\frac{\pi^{2}}{4}\right)+\pi \tan ^{-1}\left(\frac{2 x}{\pi}\right)\right) d x=\frac{1}{2} \pi\left(4 G+\pi \log \left(\frac{\pi}{2}\right)\right)
$$


Proof. Use Equation (10), set $p=2 m$, and simplify in terms of the Hurwitz zeta function $\zeta(s, v)$ using entry $(4)$ in the table below (64:12:7) in [10]. Next, we take the first partial derivative with respect to $k$, set $k=1, a=-i, m=1$, and simplify in terms of Catalan's constant $G$ after expanding in terms of the real and imaginary parts.

Lemma 12. For $\operatorname{Re}(m)>0$,

$$
\begin{aligned}
& \int_{0}^{\infty} \operatorname{csch}(m x)\left((\log (a)+x)^{k}-(\log (a)-x)^{k}\right) d x \\
& =\frac{i \pi\left(\log ^{k}(a)+2^{k+1} \pi^{k}\left(\frac{i}{m}\right)^{k}\left(\zeta\left(-k, 1-\frac{i m \log (a)}{2 \pi}\right)-\zeta\left(-k, \frac{\pi-i m \log (a)}{2 \pi}\right)\right)\right)}{m}
\end{aligned}
$$

Proof. Use Equation (10), set $p \rightarrow 2 m$, and simplify in terms of the Hurwitz zeta function $\zeta(s, v)$ using entry $(4)$ in the table below (64:12:7) in [10].

Lemma 13.

$$
\begin{gathered}
\int_{0}^{\infty} \frac{1}{4 \pi\left(x^{2}+\pi^{2}\right)} x \sinh (x) \operatorname{csch}^{3}\left(\frac{x}{2}\right)\left((\pi-i x)(-x+i \pi)^{k}(1+k \log (-x+i \pi))\right. \\
\left.-(\pi+i x)(x+i \pi)^{k}(1+k \log (x+i \pi))\right) d x \\
=\frac{1}{8} e^{\frac{i \pi k}{2}} \pi^{k-1}\left(k \left(2 i \left(4^{k+1} k \zeta^{\prime}\left(1-k, \frac{1}{4}\right)-4^{k+1} k \zeta^{\prime}\left(1-k, \frac{3}{4}\right)\right.\right.\right. \\
\left.-4^{k}(k-1)\left(\zeta^{\prime}\left(2-k, \frac{1}{4}\right)-\zeta^{\prime}\left(2-k, \frac{3}{4}\right)\right)+8 \log (\pi)\right) \\
\left.+4^{k} \zeta\left(2-k, \frac{3}{4}\right)(\pi(k-1)-2 i((k-1) \log (4 \pi)+2))\right) \\
+2\left(i 4^{k} \zeta\left(2-k, \frac{3}{4}\right)-4 \pi k+8 i\right)+4^{k+1} k \zeta\left(1-k, \frac{1}{4}\right)(k(\pi-2 i \log (4 \pi))-4 i) \\
-4^{k+1} k \zeta\left(1-k, \frac{3}{4}\right)(k(\pi-2 i \log (4 \pi))-4 i) \\
\left.-4^{k} \zeta\left(2-k, \frac{1}{4}\right)(\pi(k-1) k-2 i k((k-1) \log (4 \pi)+2)+2 i)\right)
\end{gathered}
$$

Proof. Use Equation (35), take the first partial derivatives with respect to $a, m$ and $k$, then set $a=-1, m=1 / 2$, and simplify.

\section{Example 8.}

$$
\int_{0}^{\infty} \frac{x^{2} \sinh (x) \operatorname{csch}^{3}\left(\frac{x}{2}\right)}{x^{2}+\pi^{2}} d x=8 G-4
$$

Proof. Use Equation (36), apply l'Hôpital rule as $k \rightarrow 0$, and simplify in terms of Catalan's constant $G$.

\section{Example 9.}

$$
\int_{0}^{\infty} \frac{x^{2} \sinh (x) \operatorname{csch}^{3}\left(\frac{x}{2}\right)}{4 x^{2}+\pi^{2}} d x=\frac{1}{16}\left(-32+\psi^{(1)}\left(\frac{1}{8}\right)-\psi^{(1)}\left(\frac{5}{8}\right)\right)
$$

Proof. Use Equation (35), take the first partial derivative with respect to $a, m$ and $k$, and then set $a=i, m=1 / 2$. Next, apply l'Hôpital rule as $k \rightarrow 0$ and simplify in terms of the polygamma function $\psi^{n}(z)$ using Equation (64:4:1) in [10].

\section{Example 10.}

$$
\int_{0}^{\infty} \frac{x^{2} \operatorname{coth}(x) \operatorname{csch}(x)}{4 x^{2}+\pi^{2}} d x=\frac{1}{4}(2 G-1)
$$

Proof. Use Equation (35), take the first partial derivative with respect to $a, m$ and $k$ and then set $a=i, m=1$. Next, apply l'Hôpital rule as $k \rightarrow 0$ and simplify in terms of Catalan's constant $G$ using Equations (1:7:4) and (64:3:5) in [10]. 


\section{Example 11.}

$$
\int_{0}^{\infty} \frac{x^{2} \operatorname{coth}\left(\frac{x}{3}\right) \operatorname{csch}\left(\frac{x}{3}\right)}{4 x^{2}+\pi^{2}} d x=\frac{1}{32}\left(\zeta\left(2, \frac{1}{12}\right)-\zeta\left(2, \frac{7}{12}\right)-72\right)
$$

Proof. Use Equation (35), take the first partial derivative with respect to $a, m$ and $k$, and then set $a=i, m=1 / 3$. Next, apply l'Hôpital rule as $k \rightarrow 0$ and simplify.

Example 12.

$$
\int_{0}^{\infty} \frac{x^{2} \operatorname{coth}\left(\frac{x}{3}\right) \operatorname{csch}\left(\frac{x}{3}\right)}{x^{2}+\pi^{2}} d x=\frac{1}{4}\left(-18+\psi^{(1)}\left(\frac{1}{6}\right)-\psi^{(1)}\left(\frac{2}{3}\right)\right)
$$

Proof. Use Equation (35), take the first partial derivative with respect to $a, m$ and $k$, and then set $a=-1, m=1 / 3$. Next, apply l'Hôpital rule as $k \rightarrow 0$ and simplify in terms of the polygamma function $\psi^{(n)}(z)$ using Equation (64:4:1) in [10].

\section{Example 13.}

$$
\int_{0}^{\infty} x \arctan \left(\frac{x}{\pi}\right) \operatorname{coth}\left(\frac{2 x}{3}\right) \operatorname{csch}\left(\frac{2 x}{3}\right) d x=-\frac{3}{8} \pi \log \left(\frac{27 e^{3-\frac{4 \pi}{\sqrt{3}}} \Gamma\left(-\frac{1}{6}\right)^{6}}{256 \Gamma\left(-\frac{2}{3}\right)^{6}}\right)
$$

Proof. Use Equation (35), take the first partial derivative with respect to $a, m$ and $k$, and then set $a=-1, m=2 / 3$. Next, apply l'Hôpital rule as $k \rightarrow 0$ and simplify in terms of the $\log$-gamma function $\log (\Gamma(x))$ using Equation (64:10:2) in [10].

\section{Example 14.}

$$
\int_{0}^{\infty} \frac{x(x \operatorname{coth}(x)-1) \operatorname{csch}(x)}{\left(x^{2}+\pi^{2}\right)^{3}} d x=\frac{7}{960}-\frac{18 \zeta(3)+\pi^{2}}{48 \pi^{4}}
$$

Proof. Use Equation (35), set $k=-2$, replace $a \rightarrow e^{a i}$, and simplify in terms of the polygamma function $\psi^{(1)}(z)$. Next, we form a second equation by taking the first partial derivative with respect to $a$ and simplify. Next, we take the difference of these two equations, set $a=\pi, m=1$, and simplify in terms the zeta function $\zeta(s)$ using Equation $(44: 12: 5)$ in [10]. In this evaluation, we replaced $a \rightarrow e^{a i}$ to obtain a complex number in the denominator when we evaluate and simplify the integral.

\section{Example 15.}

$$
\int_{0}^{\infty} \frac{x(x \operatorname{coth}(x)-1) \operatorname{csch}(x)}{\left(4 x^{2}+\pi^{2}\right)^{3}} d x=-\frac{512 C+32 \pi^{3}-\psi^{(3)}\left(\frac{1}{4}\right)+\psi^{(3)}\left(\frac{3}{4}\right)}{4096 \pi^{4}}
$$

Proof. Use Equation (35), set $k=-2, a=e^{a i}$, and simplify in terms of the polygamma function $\psi^{(1)}(z)$. Next, we form a second equation by taking the first partial derivative with respect to $a$ and simplify. Next, we take the difference of these two equations, set $a=\pi / 2, m=1$, and simplify in terms of Catalan's constant and the polygamma function $\psi^{3}(s)$ using Equation (44:12:5) in [10]. 


\section{Table of Definite Integrals}

Definite integrals are presented in Table 1.

Table 1. Table of definite integrals.

\begin{tabular}{|c|c|}
\hline$f(x)$ & $\int_{0}^{\infty} f(x) d x$ \\
\hline $\operatorname{csch}\left(\frac{p x}{2}\right) \sinh \left(m x-\frac{p x}{2}\right)$ & $-\frac{\pi \cot \left(\frac{\pi m}{p}\right)}{p}$ \\
\hline$x \operatorname{csch}\left(\frac{p x}{2}\right) \cosh \left(m x-\frac{p x}{2}\right)$ & $\frac{\pi^{2} \csc ^{2}\left(\frac{\pi m}{p}\right)}{p^{2}}$ \\
\hline$\frac{\log (a)\left(e^{x(p-m)}-e^{m x}\right)+x\left(e^{x(p-m)}+e^{m x}\right)}{\left(p^{p x}-1\right)\left(x^{2}-\log ^{2}(a)\right)}$ & $e^{\frac{2 i \pi m}{p}} \Phi\left(e^{\frac{2 i m \pi}{p}}, 1,1-\frac{i p \log (a)}{2 \pi}\right)+\frac{i \pi}{p \log (a)}$ \\
\hline$\frac{x^{k} e^{m x}-(-x)^{k} e^{x(p-m)}}{e^{p x}-1}$ & $\frac{i(2 \pi)^{k+1}\left(\frac{i}{p}\right)^{k} \operatorname{Li}_{-k}\left(e^{\frac{2 i m \pi}{p}}\right)}{p}$ \\
\hline$\frac{x \operatorname{csch}(x)}{x^{2}+\pi^{2}}$ & $\log (2)-\frac{1}{2}$ \\
\hline$\frac{x^{2} \operatorname{coth}(x) \operatorname{csch}(x)}{x^{2}+\pi^{2}}$ & $\frac{1}{12}\left(\pi^{2}-6\right)$ \\
\hline$\frac{x \operatorname{csch}(x)}{4 x^{2}+\pi^{2}}$ & $\frac{1}{8}(\pi-2)$ \\
\hline$\frac{x^{2} \operatorname{coth}(x) \operatorname{csch}(x)}{4 x^{2}+\pi^{2}}$ & $\frac{1}{4}(2 G-1)$ \\
\hline $\operatorname{arctanh}\left(\frac{x}{a}\right) \operatorname{csch}(m x)$ & $-\frac{i \pi \log \left(-\frac{i a m \Gamma\left(-\frac{i a m}{2 \pi}\right)^{2}}{2 \pi \Gamma\left(\frac{\pi-i a m}{2 \pi}\right)^{2}}\right)}{2 m}$ \\
\hline$x^{k} \operatorname{csch}(m x)$ & $\left(2^{k+1}-1\right) \pi^{k+1} m\left(\frac{i}{m}\right)^{k+2} \zeta(-k)\left(\cot \left(\frac{\pi k}{2}\right)-i\right)$ \\
\hline$\frac{x^{2} \operatorname{coth}(x) \operatorname{csch}(x)}{a^{2}+x^{2}}$ & $\frac{\pi}{2 a}+\frac{a\left(\psi^{(1)}\left(\frac{a}{2 \pi}+1\right)-\psi^{(1)}\left(\frac{a+\pi}{2 \pi}\right)\right)}{4 \pi}$ \\
\hline$\left(\left(x+\frac{i \pi}{m}\right)^{k}-\left(-x+\frac{i \pi}{m}\right)^{k}\right) \operatorname{csch}(m x)$ & $\frac{\pi^{k+1}\left(\frac{i}{m}\right)^{k-1}\left(\left(2^{k+2}-2\right) \zeta(-k)+1\right)}{m^{2}}$ \\
\hline$\frac{x \operatorname{csch}(m x)}{m^{2} x^{2}+\pi^{2}}$ & $\frac{\log (4)-1}{2 m^{2}}$ \\
\hline$\frac{x \operatorname{csch}(m x)}{\left(m^{2} x^{2}+\pi^{2}\right)^{2}}$ & $\frac{\pi^{2}-6}{24 \pi^{2} m^{2}}$ \\
\hline$\frac{(\pi-x)(x+\pi) \operatorname{sech}(x)}{\left(x^{2}+\pi^{2}\right)^{2}}$ & $\frac{4-4 G}{\pi}$ \\
\hline$\frac{x \operatorname{csch}(x)}{\left(x^{2}+\pi^{2}\right)^{2}}$ & $\frac{1}{24}-\frac{1}{4 \pi^{2}}$ \\
\hline
\end{tabular}

Author Contributions: Conceptualization, R.R.; writing—original draft preparation, R.R.; writingreview and editing, R.R.; supervision, A.S.; funding acquisition, A.S. Both authors have read and agreed to the published version of the manuscript.

Funding: This research is supported by NSERC Canada under Grant 504070.

Data Availability Statement: Data is contained within the article.

Conflicts of Interest: The authors declare no conflict of interest.

\section{References}

1. Olver, F.W.J.; Daalhuis, A.B.; Lozier, D.W.; Schneider, B.I.; Boisvert, R.F.; Clark, C.W.; Miller, B.R.; Saunders, B.V.; Cohl, H.S.; McClain, A.M. (Eds.) NIST Digital Library of Mathematical Functions. Available online: http:/ /dlmf.nist.gov (accessed on 8 May 2012).

2. Barr, G.E. A note on integrals involving parabolic cylinder functions. SIAM J. Appl. Math. 1968, 16, 71-74. [CrossRef]

3. Bartky, W. Numerical calculation of a generalized complete elliptic integral. Rev. Mod. Phys. 1938, 10, 264-269. [CrossRef]

4. Becker, P.A. Normalization integrals of orthogonal Heun functions. J. Math. Phys. 1997, 38, 3692-3699. [CrossRef]

5. Bronski, J.C.; Carr, L.D.; Deconinck, B.; Kutz, J.N.; Promislow, K. Stability of repulsive Bose-Einstein condensates in a periodic potential. Phys. Rev. E 2001, 63, 1-11. [CrossRef] [PubMed] 
6. Bushell, P.J. On a generalization of Barton's integral and related integrals of complete elliptic integrals. Math. Proc. Camb. Philos. Soc. 1987, 101, 1-5. [CrossRef]

7. Reynolds, R.; Stauffer, A. A Method for Evaluating Definite Integrals in Terms of Special Functions with Examples. Int. Math. Forum 2020, 15, 235-244. [CrossRef]

8. Gradshteyn, I.S.; Ryzhik, I.M. Tables of Integrals, Series and Products, 6th ed.; Academic Press: Cambridge, MA, USA, 2000.

9. Abramowitz, M.; Stegun, I.A. (Eds.) Handbook of Mathematical Functions with Formulas, Graphs, and Mathematical Tables, 9th ed.; Dover: New York, NY, USA, 1982.

10. Oldham, K.B.; Myland, J.C.; Spanier, J. An Atlas of Functions: With Equator, the Atlas Function Calculator, 2nd ed.; Springer: New York, NY, USA, 2009.

11. Adell, J.A. Estimates of generalized Stieltjes constants with a quasi-geometric rate of decay. Proc. R. Soc. A 2012. [CrossRef]

12. Guillera, J.; Sondow, J. Double integrals and infinite products for some classical constants via analytic continuations of Lerch's transcendent. Ramanujan J. 2008, 16, 247-270. [CrossRef] 